\title{
The effect of age on visuo-spatial short-term memory in family dogs
}

\author{
Patrizia Piotti*, Dóra Szabó, Lisa Wallis, Zsófia Bognár, Bianka Stiegmann, \\ Anna Egerer, Pauline Marty, Enikő Kubinyi
}

Abstract

Decline in the visuo-spatial memory domain may be an early marker for cognitive decline and has a relevant impact on animal welfare. Current research on visuo-spatial memory in family dogs is often limited by factors such as the need of extensive pre-training, limited attention to co-occurring medical conditions, a focus on laboratory dogs, or low sample size. Therefore, we aimed to develop a test that relies on visuospatial short-term memory, may be performed in a short time, and does not require explicit training. We tested a large sample of young and old dogs, finding that young dogs were more likely to perform correctly, although performance decreased with consecutive trials in both age groups. However, groups did not vary in the severity of mistakes. This task represents the first measure of dogs' age-related decline of short-term spatial memory that does not require explicit training. The test could potentially be used in veterinary behaviour contexts to monitor cognitive changes in ageing dogs, utilizing a simple binary measure of success.

Pet Behaviour Science | 2017, Vol.4, 17 - 19

DOI: $10.21071 / p b s . v 0 i 4.10130$

Patrizia Piotti, Dóra Szabó, Lisa Wallis, Zsófia Bognár, Bianka Stiegmann, Anna Egerer, Pauline Marty, and Enikő Kubinyi

ELTE Eötvös Loránd University, Pázmány Péter sétány 1/c, 1117

*Email: patrizia.piotti@yahoo.it

Budapest, Hungary

Keywords:

dog, cognitive ageing, visuo-spatial memory

This paper is based on a communication presented at '2017 Open Conference I Pet Behaviour Science' by the authors.

Please, visit https://goo.gl/6y7vJD for the prezi presentation

\section{Introduction}

Ageing in dogs is associated with the decline of several cognitive domains, such as learning, memory, visuospatial function, executive function, and attention (Folstein et al. 1975; Head 2014; Landsberg et al. 2012; Mongillo et al. 2013; Svicero \& Amorim 2017; Wallis et al. 2014). The visuo-spatial memory domain is particularly interesting, because its decline precedes the onset of declines in other domains. Therefore, visuo-spatial memory decline may be an early marker of ageing (Head et al. 1995). Furthermore, the capacity to acquire and recall the spatial features of a novel location is critical for adaptation to the environment, and impaired spatial ability can have a great impact on quality of life with advancing age. However, previous research on visuo-spatial function decline in dogs are affected by several limitations e.g. 1) testing required extensive training and lengthy protocols; 2) lack of control for potentially confounding medical conditions, such as sensory-motor impairment; 3) subject selection was limited to the laboratory dog population, which differs from non-laboratory dogs in behaviour patterns and response to social stimuli; 4) low sample sizes 
(Halmágyi 2010; Lazarowski \& Dorman 2015; Szabó et al. 2016; Wallis et al. 2014). For these reasons, the use of existing paradigms is limited to laboratory settings, and provides little benefit to the non-laboratory canine population. We therefore designed a novel task (part of a larger battery of tests), which did not require any explicit training of the dog and could be performed in the time span of a few minutes, and tested a large sample of non-laboratory dogs.

\section{Methods}

We were interested in measuring whether the task could detect age-dependent short-term memory changes, thus, we compared two groups of dogs, based on their age; 'young $\operatorname{dogs}^{\prime}\left(\mathrm{N}=44, \mathrm{Mdn}_{\text {age }}=4.6\right.$ years, $\mathrm{IQR}=3.3-6.0$, Females $=61 \%)$, and 'old dogs' $(\mathrm{N}=75$, $\mathrm{Mdn}_{\text {age }}=10.7$ years, IQR $=9.0-11.6$, Females $=43 \%$ ). Dogs of various breed types, medium to large sized, were recruited through an online survey. Before testing, the dogs' sensory-motor function was assessed in a standardised way, in order to exclude conditions that could potentially impair the dogs' performance during testing. The tests were performed in an empty room, where 5 identical containers were positioned on the floor equidistant from each other at regular intervals along a semi-circle so that they were all equally distant from a pre-determined location 2 meters away. At the beginning of each trial, the owner stood at the predetermined location and held the dog by the leash. The dog witnessed an experimenter baiting 1 of the 5 identical plastic containers and was then walked out of the room. After a 30 seconds distraction task (petting or playing with the dog), the dog was walked back into the room, was let off leash and was allowed to approach the containers. The experimenter recorded the first choice made by the dog and the severity of mistakes the dog made. The test was repeated once per container; the order of baited locations was counterbalanced across participants.

\section{Results}

Two linear models were calculated to analyse the effect of age group, breed, and sex on the measured variables. For each model, an automated model selection process generated multiple models with combinations of these factors; models estimating both intercept and/or slope for random effects were also calculated. For each response variable, the model that had the lowest Akaike information criterion (AIC) score, as tested by likelihood ratio test, was then selected. A generalised linear mixed model (GLMM) fit with maximum likelihood approximation, with binomial error structure and logit link function was selected for the response variable 'first approach' (correct vs incorrect), with the random intercept factor 'dog identity'. A main effect without interaction of the fixed factors 'age group' (young or old) and 'trial number' (1 to 5 ) on the outcome variable was observed $\left(\mathrm{GLMM}_{\mathrm{AgeGroup}+\mathrm{Trial}(\mathrm{Dog}),}\right.$ $\mathrm{AIC}=745.04, \mathrm{~N}=595$, number of subjects $=119$, $\left.\chi_{4}=14.66, p=0.001\right)$. Young dogs were more likely to choose the correct container compared to the old dogs (Post-hoc Tukey: estimate Young-Old $\pm \mathrm{SE}=0.627 \pm 0.222$, $\mathrm{p}=0.005)$, and dogs in both groups were overall less likely to choose the correct container as trials progressed (estimate Trial $\pm \mathrm{SE}=-0.165 \pm 0.06, \mathrm{p}=0.011$ ) . For the response variable 'mistakes' (scores from 0 to 3 , with $0=$ most severe mistakes; $3=$ no mistakes), the lowest AIC was yielded by another GLMM estimated by maximum likelihood with Poisson error structure and log link function, with the nested random intercept factors 'dog identity' and 'trial' and a main effect for the fixed factor 'age group' . However, the model was not significantly different from the null model, i.e. including the intercept only $\left(\mathrm{GLMM}_{\mathrm{AgeGroup}}, \mathrm{AIC}=1851.2\right.$, $\mathrm{N}=595$; number of subjects $=119, \chi_{4}=2.24, \mathrm{p}=0.135$ ).

\section{Conclusion}

This task represents the first example of a protocol relying on short-term spatial memory that does not require any explicit training and identifies a difference in performance associated with age. Due to its simplicity, the task could potentially be used outside the laboratory environment, e.g. by veterinary professionals, in order to monitor cognitive changes in ageing dogs. Care is needed in the selection of the outcome variable: a binary measure might be more effective than scores based on incorrect choices. Further research is required to determine performance ranges at the population level and identify changes associated with pathological conditions affecting cognitive abilities, e.g. Canine Cognitive Dysfunction Syndrome. 


\section{Funding}

This project has received funding from the European Research Council (ERC) under the European Union's Horizon 2020 research and innovation programme (Grant Agreement No. 680040) and was supported by the János Bolyai Research Scholarship of the Hungarian Academy of Sciences for EK.

\section{Acknowledgments}

We would like to thant Sarolta Marosi, Vivien Hemzö, Szandy Deés, Frida Katona, for their help with the data collection and coding and Louis Le Nézet for his help with coding.

\section{References}

Folstein, M. F., Folstein, S. E., \& McHugh, P. R. (1975). A practical state method for. Journal of Psychiatric Research, 12(3), 189-198. http://doi.org/10.1016/00223956(75) $90026-6$

Halmágyi, E. (2010). Családi és laboratóriumi beagle kutyák személyiségének összehasonlító elemzése [Comparative analysis of the personality of family and laboratory beagle dogs] MSc Thesis. Eötvös Loránd University.

Head, E. (2014). A Canine Model of Human Aging anD Alzheimer's Disease. Biochimica et Biophysica Acta, 1832(9), 1384-1389.

http://doi.org/10.1016/j.bbadis.2013.03.016.A

Head, E., Mehta, R., Hartley, J., Kameka, M., Cummings, B. J., Cotman, C. W., ... Milgram, N. W. (1995). Spatial learning and memory as a function of age in the dog. Behavioral Neuroscience, 109(5), 851858. http://doi.org/10.1037/0735-7044.109.5.851

Landsberg, G. M., Nichol, J., \& Araujo, J. A. (2012). Cognitive Dysfunction Syndrome. A Disease of Canine and Feline Brain Aging. Veterinary Clinics of North America - Small Animal Practice, 42(4), 749-768.

http://doi.org/10.1016/j.cvsm.2012.04.003
Lazarowski, L., \& Dorman, D. C. (2015). A comparison of pet and purpose-bred research dog (canis familiaris) performance on human-guided object-choice tasks. Behavioural Processes, 110, 60-67. http://doi.org/10.1016/j.beproc.2014.09.021

Mongillo, P., Araujo, J. A., Pitteri, E., Carnier, P., Adamelli, S., Regolin, L., \& Marinelli, L. (2013). Spatial reversal learning is impaired by age in pet dogs. Age, 35(6), 2273-2282. http://doi.org/10.1007/s11357-0139524-0

Svicero, D. J., \& Amorim, R. (2017). Prevalence of behavioral changes in senile dogs. Clinic and Surgery, 47(2). http://doi.org/10.1590/0103-8478cr20151645

Szabó, D., Gee, N. R., \& Miklósi, A. (2016). Natural or pathologic? Discrepancies in the study of behavioral and cognitive signs in aging family dogs. Journal of Veterinary Behavior: Clinical Applications and Research, 11, 86-98.

http://doi.org/10.1016/j.jveb.2015.08.003

Wallis, L. J., Range, F., Müller, C. A., Serisier, S., Huber, L., \& Virányi, Z. (2014). Lifespan development of attentiveness in domestic dogs: Drawing parallels with humans. Frontiers in Psychology, 5(71), 1-13. http://doi.org/10.3389/fpsyg.2014.00071

\section{cc) creative}

This paper has been published by Pet Behaviour Science under a Creative Commons license 4.0 Non-comercial - Share Alike - Attribution

As an open access journal, it is free of charges for both authors and readers

www.petbehaviourscience.org 